\title{
SQUARE ROOT INTENSITY CODING IN TURTLE CONES: PHYSIOLOGICAL MECHANISMS
}

\author{
Vincent Pluvinage and Daniel G. Green \\ From the Vision Research Laboratory, Neuroscience Building, University of Michigan, 1103 E. Huron, \\ Ann Arbor, MI 48109, U.S.A.
}

(Received 3 November 1988; in revised form 22 September 1989)

\begin{abstract}
The effect of coupling on the intensity-response functions for full field and for slit stimuli was studied by comparing the shapes of the curves obtained from several strongly coupled red cones of turtle with that of one very weakly coupled cone. The full field $V-\log I$ curves could be fitted by a Michaelis-Menten relationship, regardless of the strength of the coupling. For the weakly coupled cone the slit $V-\log I$ could also be fitted by a Michaelis-Menten curve. For strongly coupled cones a major portion of the curve (1.2-2 log units of intensity) was better fitted by $V \propto I^{m}$. For centered slits " $m$ " was 0.5 . With increased distance between the slit and the center of the receptive field " $m$ " was found to increase slightly. The results were analyzed in terms of a theory in which the shape of the slit intensity-response curves arises from scattered light progressively recruiting neighboring cone responses. An analytical formulation of this idea is presented and a plausible light distribution function which supports the recruitment hypothesis is derived. A numerical model of the cone network, which includes the effect of scattering and transduction saturation, accounts well for all of the experimental data obtained with single and paired slits.
\end{abstract}

Retinal cone Phototransduction Photoreceptor

\section{INTRODUCTION}

In our previous paper (Pluvinage \& Green, 1990), we used intracellular responses from turtle cones to infer the relationship between the intensity of a small light stimulus and the amplitude of its local neural correlate, which we called excitation. Over two log-units of intensity, excitation was found to be approximately proportional to the square root of the light intensity, a result consistent with the intensity coding inferred from ganglion cell studies in other animals (Easter, 1968; Levine \& Abramov, 1975; Enroth-Cugell \& Harding, 1980). The aim of this paper is to clarify the physiological mechanisms which lead to the 'square root' relationship.

We first present experimental evidence that the "square root" relationship is intimately related to photoreceptor coupling. This leads to the following idea about how the square root relationship arises. As the intensity of a slit stimulus centered on an impaled cone is increased, two things happen. The cones in the geometric image of the slit saturate, and increasing numbers of coupled cones are stimulated by scattered light. The square root relationship for small stimuli results from local saturation of cones and from the summation of signals from an ever increasing area of the retina. The evidence for this idea comes from a model incorporating light scattering, a Michaelis-Menten photocurrent transduction, and electrical coupling between cones. Within the model, recruitment of coupled cone responses can account for all of the experimental findings.

\section{MITHODS}

The methods are those described in the previous paper (Pluvinage \& Green, 1990).

\section{RXSULTS}

The effect of coupling on the intensity-response curves

The effects of photoreceptor coupling are well illustrated by comparing the intensity-response curves of a strongly coupled cone and a very weakly coupled one. According to Baylor and Hodgkin (1973) and Lamb and Simon (1976) the strength of the coupling is proportional to the receptive field space constant measured with a narrow slit of light. Figure 1 compares the receptive field profiles for two cells with very different space constants. The profile of a 


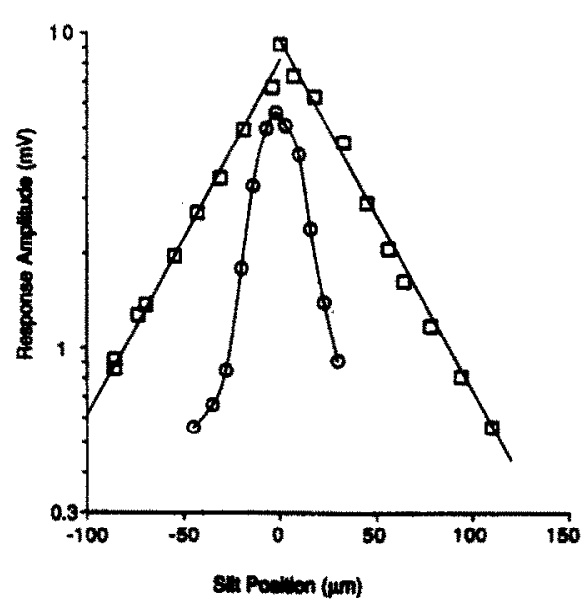

Fig. 1. Receptive field profiles for two cones. The data points indicate the peak amplitude of responses to a slit stimulus flashed at various distances from the center of the recoptive field. The receptive field of a strongly coupled cone (D) is well fitted by exponential fall of, as shown by the repreation lines on logarithmic coordinutes (colid lines). The receptive field of a weakly coupled cone $(O)$ is not adequately fitted by exponential fall off (the line was drawn by eye).

strongly coupled cone ( $\square$ ) was fitted by linear regression (solid lines) on semi-logarithmic coordinates. The space constants of the exponentials are 29 and $33 \mu \mathrm{m}$ (left and right sides respectively). The profile of a weakly coupled cone $(O)$ was not adequately fitted by exponential functions (the dached curve was drawn by eye). The steepest seyments of the profile have slopes which correspond to space constants of 10 and $12 \mu \mathrm{m}$ respectively.

For both cones, we systematically measured the peak amplitude of the responses to full field and slit stimuli flashed over a wide range of intensities. Figure 2 shows the intensityresponse curves for the strongly coupled cone. The data for the full field stimuli $(O)$ were fitted by a Michaelis-Menten relationship:

$$
\frac{V}{V_{\max }}=\frac{I}{I+\sigma}
$$

where $V$ is the peak amplitude (in $\mathrm{mV}$ ), $\mathrm{V}_{\max }$ is the saturating amplitude, $I$ is the light intensity, and $\sigma$ is the intensity which produces a halfmaximum response. The data for the slit stimulus $(O)$ were obtained for several positions of the slit with respect to the receptive field center. Over 1.3-1.6 log units of intensity a straight line in double logarithmic coordinates fits each set of data points reasonably well, indicating a power law relationship. This is in agreement with the analysis presented in the previous paper (Pluvinage \& Green, 1990). The slope of the straight lines gives the exponent of the power law. For this cell, it varies from 0.54 for the

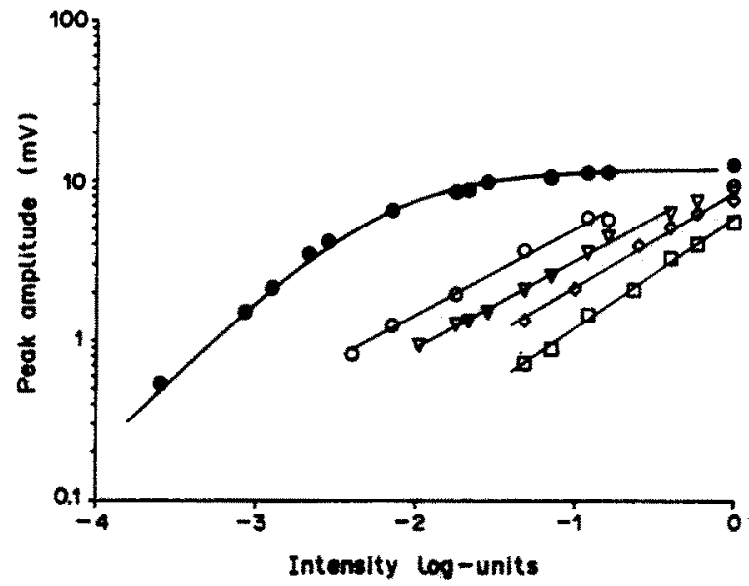

Fig. 2. Intensity-response curves for a stronity cownded cone (anme as in Fig. 1). The full fuld dats ( $S$ apol diameter: $170 \mathrm{\mu m}$ ) were fitted by a Miahedim-Menten relationship (solid curve; $V_{\max }=12 \mathrm{mV}, X=-2.3$ ). The slit date (open symbola) were fitted by power lows (stringt lines). Stit position on the receptive fild: $\square,-50 ; 0,+40 ; \nabla,-25$; $0,0 \mu \mathrm{m}$. The power law exponents (slope of the regreation lines in logarithmic coordinates) are given in Table 1.

centered slit to 0.68 for a slit displacement of $50 \mu \mathrm{m}$ (see Table 1). This increase in the exponent with slit displacement was observed

Table 1. Summary of the intensity-response curves. Stit position refers to the location of the alit (in $\mu \mathrm{m}$ ) relative to the center of the recoptive field, " + " indicating one tiste end

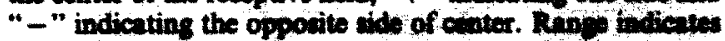
the number of los units of intentity over which a etralyt line in donble lo vithmic coonthetes fited the data. The $r^{2}$ is the regresion coeficient for the power law which geve the best fit

\begin{tabular}{|c|c|c|c|c|}
\hline $\begin{array}{l}\text { Cell } \\
\text { no. }\end{array}$ & $\begin{array}{c}\text { Slit } \\
\text { position }\end{array}$ & $\begin{array}{l}\text { Power law } \\
\text { exponent }\end{array}$ & Range & $r^{2}$ \\
\hline 1 & $\begin{array}{r}0 \\
+30\end{array}$ & $\begin{array}{l}0.45 \\
0.53\end{array}$ & $\begin{array}{l}1.2 \\
1.2\end{array}$ & $\begin{array}{l}0.97 \\
0.96\end{array}$ \\
\hline 6 & $\begin{array}{r}0 \\
+30 \\
+50\end{array}$ & $\begin{array}{l}0.5 \\
0.51 \\
0.66\end{array}$ & $\begin{array}{l}2.0 \\
1.2 \\
1.0\end{array}$ & $\begin{array}{l}0.96 \\
0.96 \\
0.99\end{array}$ \\
\hline 7 & $\begin{array}{r}0 \\
+40 \\
+60\end{array}$ & $\begin{array}{l}0.45 \\
0.49 \\
0.55\end{array}$ & $\begin{array}{l}1.4 \\
1.0 \\
1.0\end{array}$ & $\begin{array}{l}0.97 \\
0.99 \\
0.96\end{array}$ \\
\hline 8 & $\begin{array}{r}0 \\
-30 \\
-40 \\
-50 \\
-63\end{array}$ & $\begin{array}{l}0.46 \\
0.48 \\
0.48 \\
0.49 \\
0.57\end{array}$ & $\begin{array}{l}2.0 \\
1.5 \\
1.8 \\
2.0 \\
1.0\end{array}$ & $\begin{array}{l}0.99 \\
0.99 \\
0.99 \\
0.99 \\
0.99\end{array}$ \\
\hline 9 & $\begin{array}{r}0 \\
-15 \\
+15 \\
-30 \\
+30 \\
+40 \\
-50 \\
+60\end{array}$ & $\begin{array}{l}0.53 \\
0.53 \\
0.55 \\
0.55 \\
0.59 \\
0.63 \\
0.58 \\
0.7\end{array}$ & $\begin{array}{l}1.6 \\
1.8 \\
2.0 \\
2.0 \\
1.8 \\
1.4 \\
1.5 \\
1.0\end{array}$ & $\begin{array}{l}0.98 \\
0.98 \\
0.99 \\
0.99 \\
0.99 \\
0.98 \\
0.96 \\
0.92\end{array}$ \\
\hline 10 & $\begin{array}{r}0 \\
-25 \\
+25 \\
+40 \\
-50\end{array}$ & $\begin{array}{l}0.54 \\
0.54 \\
0.5 \\
0.59 \\
0.68\end{array}$ & $\begin{array}{l}1.6 \\
1.5 \\
1.3 \\
1.3 \\
1.3\end{array}$ & $\begin{array}{l}0.99 \\
0.99 \\
0.99 \\
0.99 \\
0.99\end{array}$ \\
\hline
\end{tabular}




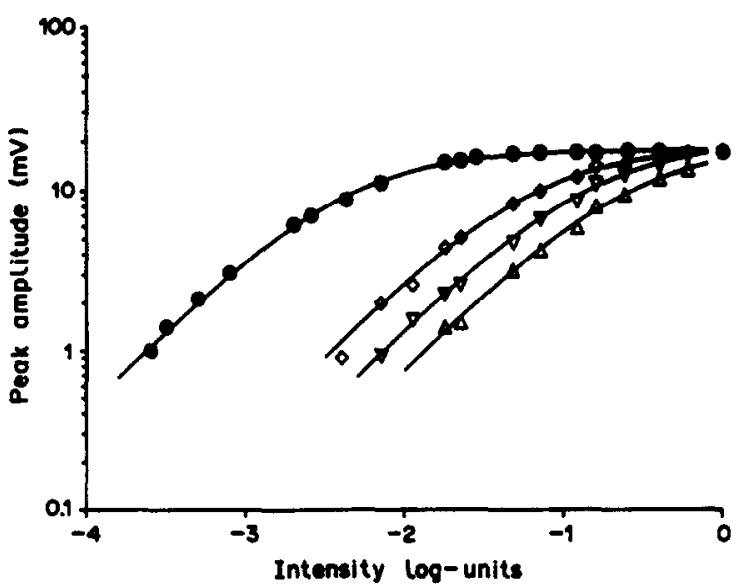

Fig. 3. Intensity-response curves for a weakly coupled cone (same as in Fig. 1). Both the full field (O) and slit (open symbols) data were fitted by Michaelis-Menten relationships. Slit positions: $\vartheta,+10 ; \nabla,-17 ; \Delta,+23 \mu \mathrm{m}$ from the center of the receptive field.

consistently in other cells as well (see Table 1). The responses saturated at high intensities, as indicated by the curved solid line drawn through the upper data points of each curve. Those data points were excluded from the power law fit.

Figure 3 shows the same measurements for the weakly coupled cone. Here, both the full field data points $(O)$ and the slit data $(0, \Delta, \nabla)$ were adequately fit by Michaelis-Menten intensity-response relationships (equation 1). All the sets of data points have virtually the same $V_{\text {max }}(18.0 \mathrm{mV})$ and differ only by the value of $\sigma$ (values obtained by regression: full field: -2.4 ; slit, position $+10 \mu \mathrm{m}$ : -1.21 ; position $-17 \mu \mathrm{m}:-0.85$; position $+23 \mu \mathrm{m}:-0.62 \log$

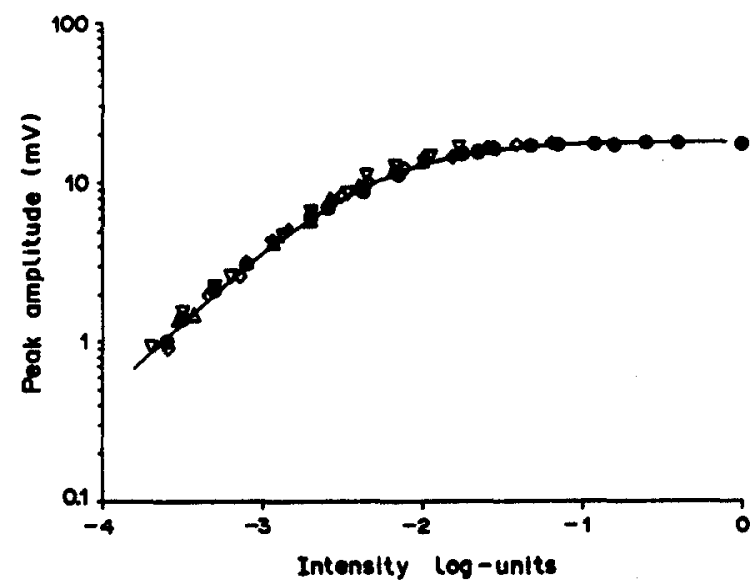

Fig. 4. Same data as in Fig. 3 (weakly couple cone), but the slit data were shifted horizontally to illustrate that the Michaelis-Menten relationships differ only by the value of the constant $\sigma$. For each slit potition the amount of horizontal shift was such that the fitted curves superimposed at half maximum amplitude.

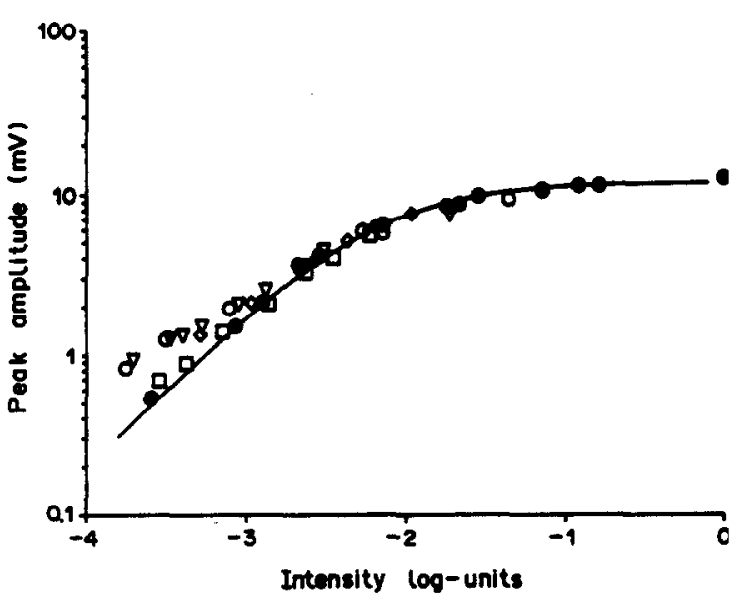

Fig. 5. Same data as in Fig. 2, (strongly coupled cone) but the slit data were shifted horizontally until they superimposed at half maximum amplitude. This indicates clearly that the slit data for the cone are not adequately fitted by a Michaelis-Menten relationship (solid line).

units). This is illustrated on Fig. 4 by shifting the slit curves horizontally until they superimpose with the full field curve. This is in contrast with the data obtained from the strongly coupled cone. As seen in Fig. 5, when the slit data are shifted horizontally, a single Michaelis-Menten curve fails to fit all the points.

When a spatially restricted stimulus such as a narrow slit is used, a strongly coupled cone and a weakly coupled cone have intensity-response curves that differ in shape. For the weakly coupled cone most of the response is due to light falling directly on the impaled cell's outer segment and the relationship is always MichaelisMenten. For the strongly coupled cone this is not the case and the measured relationship, which is approximately a power law, must reflect some property of the coupling between cones.

\section{Model of the recruitment mechanism}

How might the power law relationships be due to the summation of signals from non-uniformly illuminated coupled cones? For a centered slit the coupled receptors are stimulated directly by the slit and indirectly by scattered light. If photocurrent in each cone is a Michaelis-Menten function of intensity (Schnapf \& McBurney, 1980), then the cones illuminated directly by a relatively bright centered slit could be partially saturated. More distant coupled cones that receive scattered light might still be in their linear range. Brighter stimuli could evoke larger responses by stimulating these yet unsaturated cones. The question which needs to be addressed is: can the amount 
of scattered light decrease with distance from the slit in such a way that the amplitude of the response to centered and displaced slits will grow with light intensity according to a power law? To answer this the network of coupled cones has to be modeled. Appendix 1 describes the details of how this was done. Appendix 2 shows that if $S(x)$, the scattered light distribution in the image of a slit, is given by:

$$
S(x)=S(0)\left[1+\gamma \lambda\left(1-\mathrm{e}^{-|x| / \lambda}\right)\right]^{-2},
$$

then the intensity response curve will be a power law with a power of 0.5 .

\section{Comparison between model simulations and data}

The full field and slit intensity-response curves were simulated numerically using a discrete array of 83 cones whose positions were obtained from a photomicrograph published by Hodgkin (1971) and the scattered light function shown in Fig. 9B. These are compared to the experimental data in Fig. 6. Three parameters were adjusted to provide the best fit: $V_{\max }$, the maximum amplitude; $\sigma$, the full field intensity which evokes half maximum amplitude; and $\delta$, the coupling space constant (see Appendix I).

The receptive field profiles were also simulated and compared with the data (Fig. 7). The response (model: solid line; data: $O$ ) and sensitivity (model: dashed line; data: $\square$ ) profiles decay approximately exponentially with markedly different slopes.

\section{Simulation of two simultaneous slits}

The model was further tested by computing the intensity-response curves for two simul-

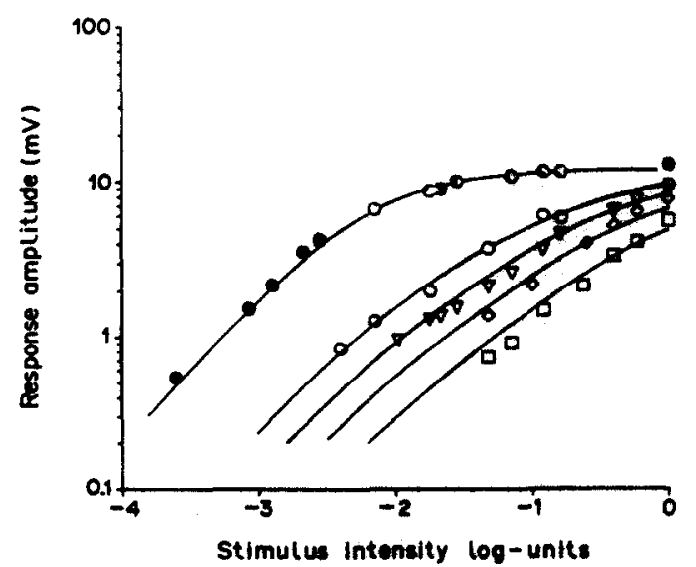

Fig. 6. Comparison between the simulation of the intensity-response curves for the discrete modal (solid lines) and the data shown in Fig. 2. The simulation procedure is given in detail in the text. The symbols used are the same as in Fig. 2.

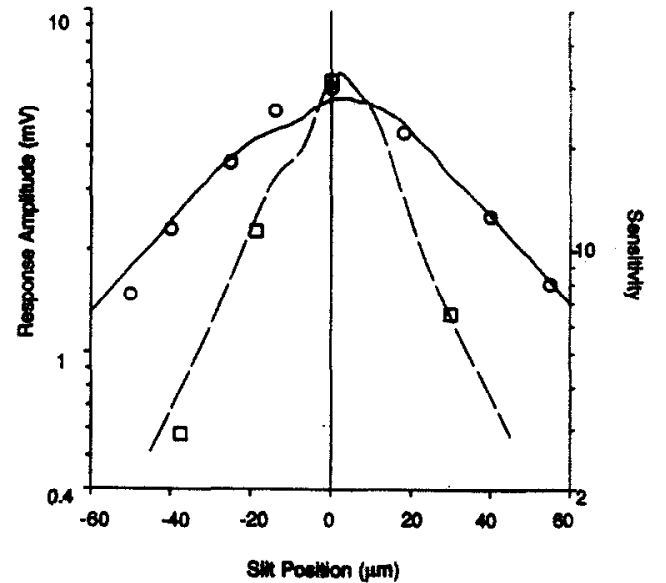

Fig. 7. Comparison between the simulated receptive field profiles and the data. The response amplitude $(O)$ to a fired intensity $(-1 \log )$ slit and the sensitivity $(\square$; fixed reponse amplitude: $3 \mathrm{mv}$ ) fall off with very different space conatents. The discrete model was used to numerically simulate both profiles using the same parameters as used to fit the intensity-response curves (response profiles: solid line; sensitivity profile: dashed line). Both decay exponentially and agree well with the data (the dight deprescion on the lof of the peak is due to a lower cone denaity in that rejion of the digitized cone array).

taneously flashed slits, one centered and one displaced by $30 \mu \mathrm{m}$. The values of the model's parameters were those used to fit the data shown in Figs 6 and 7. The intensity-response curves for each slit alone and for both slits flashed simultaneously were calculated.

An excitation function $e($.$) , was obtained by$ applying the excitation method described in Pluvinage and Green (1990) to the calculations. The simulated excitation function $e($.$) is shown$ in Fig. 8A and is compared with data from cell 1 of Pluvinage and Green (1990).

The transformation that takes the excitation variable for a centrally placed spot into the measured intracellular response is denoted $v($.$) .$ The simulated and measured $v($.$) 's are shown in$ Fig. 8B. In order to compare the simulated function with the measured function $v($. (Pluvinage \& Green, 1990) two rescalings were necessary. One rescaling takes into account the differences in amplitudes of the maximum response $(15.5 \mathrm{mV}$ for cell 1 of Pluvinage \& Green, $1990 ; 12.0 \mathrm{mV}$ for the data from the cell shown in Fig. 2 and used to determine the values of the model's parameters). The other rescaling brings the model and experimental units of excitation into registration. The simulated function shows a progressive saturation although not as pronounced as the measured function.

The additional transformation needed to 

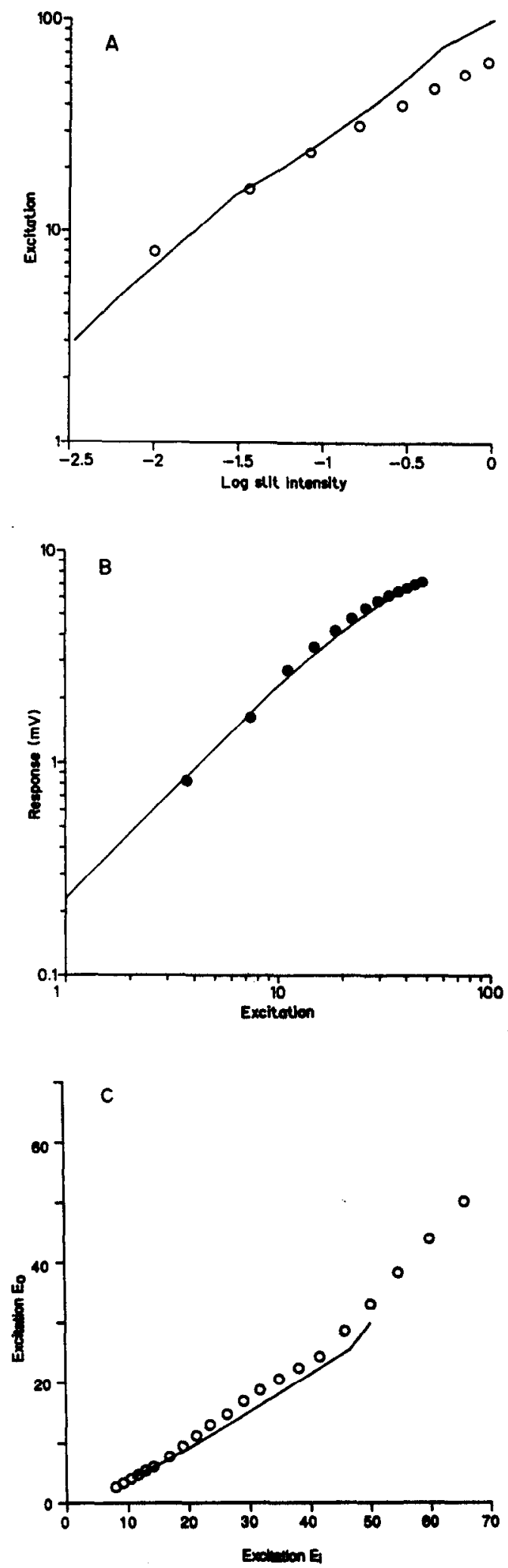

Fig. 8. Comparison of theoretical curves with experimental data. Circles obtained from measurements on cell 1 of Pluvinage and Green (1990) and continuous curve is from the two-dimensional model simulation. (A) Excitation functions, $e($.$) . (B) Output functions, v($.$) . (C) Coupling$ functions, $n($.$) .$ account for the response to the displaced spot is denoted as $n($.$) (see Fig. 2$ of Pluvinage \& Green, 1989). Finally, the measured and the simulated coupling function $n($.$) are compared$ in Fig. 8C. Both exhibit a lower range where excitation can be fitted by a straight line. That is, the excitation evoked at position 0 is a linear function of the excitation evoked $30 \mu \mathrm{m}$ away. The slopes are 0.78 and 0.61 and the intercepts are -5.3 and -3.2 for the lines fitted by linear regression to the lower portions of measured and simulated functions, respectively. Both functions deviate similarly at highest values of excitation. The second order agreement between the simulation and the data was totally unexpected and tends to give additional support to the model presented here.

\section{DISCUSSION}

Sensation frequently grows as a compressive power function of stimulus strength. A sensory receptor with such behavior would be capable of handling a considerably larger range of intensities than one with a Michaelis-Menten transduction function (see Green, 1986). Since power law responses from vertebrate photoreceptors have not been reported before, it is useful to examine in detail how this range expanding mechanism seems to work.

With a full field stimulus, all the photoreceptors in the impaled cone's receptive field would tend to be equally stimulated. There would be little or no potential gradient betwen cells and current would not flow between coupled cones. Intensity response curves for the weakly and the strongly coupled cones have identical shapes which suggests that a Michaelis-Menten relationship characterizes the response properties of the cones that are uncoupled.

This leads us to conclude that the "square root" relationship for the responses to slits must in some way be due to the contributions from coupled cones stimulated by scattered light. A quantitative model of the combined effects of scattering, transduction saturation and coupling has been used to test the notion that the power law relationship arises from a recruitment phenomena. Figure 6 shows a comparison of measured and calculated intensity-response curves. The four solid theoretical curves drawn through the experimental points (open symbols in Fig. 6) agree well with the data. It is perhaps not surprising that the intensity-response curve for 
a centered slit has the correct shape at lower intensities since scattered light distribution was selected to produce the required slope. However, the progressive saturation at high intensities, the increase in slope with slit displacement, and the position of the slit curves with respect to the full field curve constitute genuine predictions of the model.

The model was used to compute the response to a centered slit over a range of intensities. The relative contribution of the cells in $10 \mu \mathrm{m}$ wide bands on either side of the impaled cell is illustrated. At low intensities the cones lying in the 0th band (i.e. the ones having an abscissa$5 \mu \mathrm{m}<x_{i}<5 \mu \mathrm{m}$ ) contribute approx. $85 \%$ of the response, and only $15 \%$ is due to responses from light scattered to neighbors. As the intensity is increased, the cones in the central band progressively saturate because of the nonlinear Michaelis-Menten transduction function and the relative contributions from the coupled neighbors increase.

The excitation analysis presented in Pluvinage and Green (1990) suggested that a power law transformation occurs before the spatial interactions mediated by the cone network. The experiments illustrated in Figs 2 and 3 show that the shape of the intensity-response curve for a slit stimulus, but not for a full-field one, depends on the strength of the coupling. This seems to contradict this interpretation of the excitation experiment. The origin of the contradiction can be traced back to two implicit assumptions of the excitation analysis. We assumed that the narrowness of the slits was such that their responses were from a single "row" of equally stimulated photoreceptors. In fact, "excitation" reflects the properties of a collection of cones. Our analysis suggests that it is not a change in a neural signal but rather changes in the size of the collection of cells with stimulus intensity that underlies the power law intensity-response transformation. The second assumption was that two slits could be used simultaneously and independently to increase the total excitation. The scattering function used in our model has tails that extend over an appreciable distance. Consequently, at high stimulus intensities, the collection of excited cells with slits separated by $30 \mu \mathrm{m}$ would overlap significantly. Because the Michaelis-Menten transduction relationship is a compressive function, the transduction signal generated by two simultaneous slits would then be less than the sum of their individual signals. It is the nonindependence of the slit stimuli that
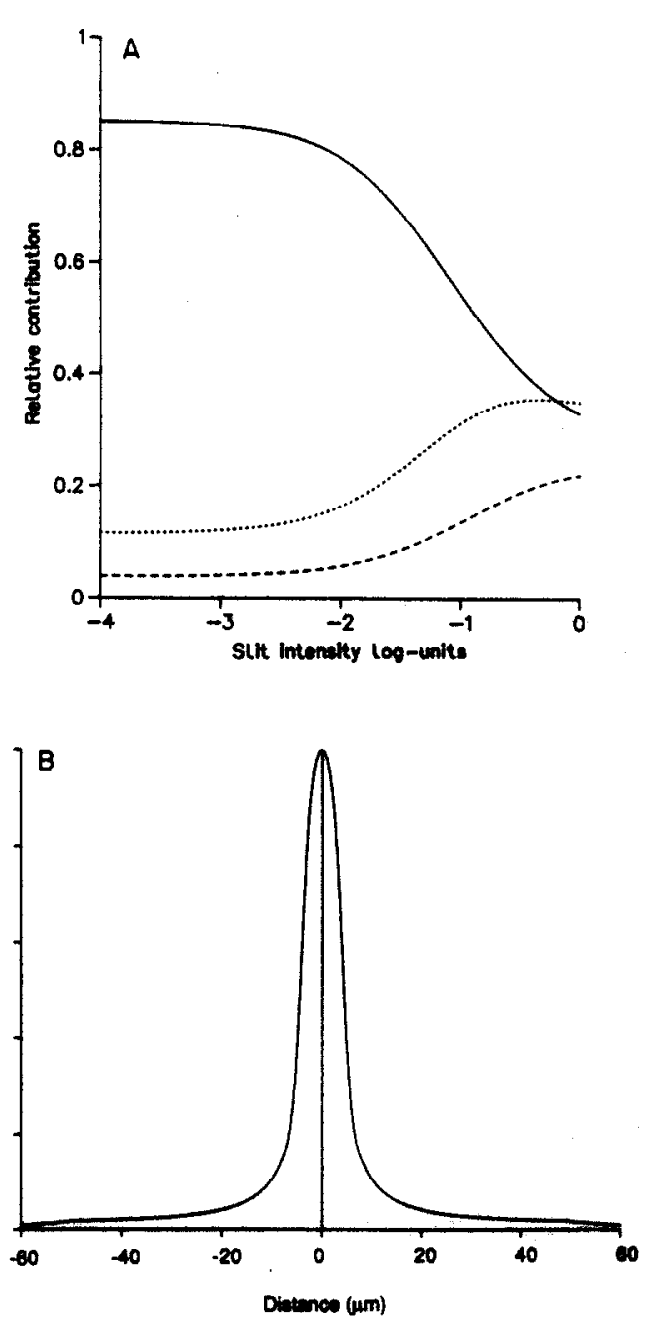

Fig. 9.(A) Effect of intensity on the relative contribution to the response from cones at various distances from the impaled photoreceptor. The cone array was divided in vertical bands, $10 \mu \mathrm{m}$ wide. The band 0 is centered on the $x$-axis (axis of the centered slit); band 1 extends from $x=+5 \mu \mathrm{m}$ to $x=+15 \mu \mathrm{m}$; etc. Symbols: solid curve $=$ band 0 , dotted curve $=$ bands 1 and -1 , dashed curve $=$ bands 2 and -2 . For each slit intensity, the discrete model was used to compute the impaled cone's responee, which is the sum of the contribution from all the bands in the cone array. (B) Light distribution in the image of a slit stimulus that was used in calculating theoretical curves shown in Figs $6-9(\gamma=1, \lambda=25)$.

leads to saturation of the output function shown in Fig. 8B.

The model fits the data but is it reasonable? The first building block, the shape of the slit distribution, $S(x)$, plays a major role in the recruitment hypothesis. The precise pattern of illumination determines the pattern of stimulation levels across the pool of cones that produce the response. This in turn determines the shape of the intensity-response curve. Before we derived the analytical solution presented in 
Appendix 2, we unsuccessfully tried several arbitrary light distributions (inverse square decay, exponential decay, etc.), none of which led to an appreciable power law range. The question that naturally arises then is: how critically does the fit of the model to the data depend on the inferred function $S(x)$ ? The power law relationship was observed in many cones in different retinas. Thus the explanation should be robust enough to maintain its main characteristics despite variations in anatomical distribution of cones, retinal scattering, coupling, strength, etc.

We believe that the concrete form of $S(x)$ is just one example of a class of scattering functions which will yield an appreciable "square root" range. Two observations are relevant here. First, although $S(x)$ depends mathematically on the strength of coupling, changing the value of $\lambda$ in equation 1.7 from 15 to $40 \mu \mathrm{m}$ has very little on the shape of $S(x)$ and of the intensity-response curves. Second, the simulation of the excitation analysis using parameters for one cell agreed surprisingly well with the results of the analysis conducted using data from another cell.

The presence in the light distribution of long, slowly decreasing "tails" extending over the whole receptive field seems to be what is important. It is noteworthy that the light distribution inferred by Copenhagen and Owen (1976) from the data obtained by Baylor and Hodgkin (1973) also has prominent "tails", extending up to $125 \mu \mathrm{m}$ away from the peak. Furthermore, the parameters in discrete model simulations correspond to a $\lambda=18 \mu \mathrm{m}$ for the coupling space constant whereas the computed receptive field profile for that cell has a $\lambda_{r}=29 \mu \mathrm{m}$. This supports Detwiler and Hodgkin's (1979) suggestion that the tail of the scattering function leads to an overestimate by approx. $50 \%$ of the coupling space constant. The above is for the eyecup preparation, thus we must ask: are these prominent "tails" in the intact eye as well? Robson and Enroth-Cugell (1978) made direct measurements of the light distribution in the cat's retinal image and found prominent "tails". This seems likely to be true for the turtle as well.

The second building block of the model, the light intensity/peak photocurrent relationship, is based on the measurements from isolated cones (Schnapf \& McBurney, 1980). The model does not incorporate time-dependent, voltage controlled components of the membrane cur- rent (as opposed to the photosensitive current) such th those metastied on tiger salamander cones by Attwell et al. (1982). These would seem to play only a small role in shaping the peak amplitude of cone voltage response to light.

The third building block, the resistive coupling, was introduced by previous investigators (Lamb \& Simon, 1976; Detwiler \& Hodgkin, 1979) and has been tested using dual recording from pairs of coupled cones and found to be essentially ohmic.

Finally, it is interesting to note that early compressive power law excitation functions with exponents of about 0.5 have been inferred from ganglion cell studies in other animals (Easter, 1968 and Levine \& Abramov, 1975: goldfish; Enroth-Cugell \& Harding, 1980: cat). It is certainly intriguing that a similar procedure applied to turtle cone responses and to fish and cat ganglion cell responses leads to a virtually identical inference about early intensity coding. While it is tempting to do so, it may be a mistake to conclude that there is any relationship between their inferences and ours. The stimuli used (spots of several hundreds of microns in diameter flashed on equally sensitive positions of the ganglion cell receptive fields) differ from those in our study. In turtle such large stimuli would extend over areas much larger than the cone receptive field. Such full field stimuli evoke intracellular responses in turtle cones which are Michaelis-Menten and not power-law functions of light intensity. Nonetheless, it still seems possible that light scatter and recruitment of locally saturating sub-units might explain the early square-root transformations inferred in these studies. Thus, the relationship, if any, between the ganglion cell studies and the present results from cones must for the moment remain unclear.

Acknowledgements - This study was supported by NIH grant EY00379. The authors are indebted to David Krantz and Stephen Easter for helpful discussions and to Marilyn Glover for her assistance in the analysis of data and in the preparation of the manuscript.

\section{REFERFNCTS}

Attwell, D., Werblin, F. S. \& Wilson, M. (1982). The properties of single cones isolated from the tiger salamander retina. Journal of Physiology, London, 328, 259-283.

Baylor, D. A. \& Hodgkin, A. L. (1973). Detection and resolution of visual stimuli by turtle photoreceptors. Joumal of Physiology, London, 234, 163-198. 
Copenhagen, D. R. \& Owen, W. G. (1976). Functional characteristics of lateral interactions between rods in the retina of the snapping turtle. Journal of Physiology, London, 259, 251-282.

Detwiler, P. B. \& Hodgkin, A. L. (1979). Electrical coupling between cones in turtie retina. Journal of Physiology, London, 291, 75-100.

Easter, S. S. (1968). Excitation in the goldfish retina: Evidence for a non-linear intensity code. Journal of Physiology, London, 195, 253-271.

Enroth-Cugell, C. \& Harding, T. H. (1980). Summation of rod signals within the receptive field center of cat retinal ganglion cells. Journal of Physiology, London, 298, $235-250$

Green, D. G. (1986). The search for the site of visual adaptation. Vision Research, 26, 1417-1429.

Hodgkin, A. L. (1971). Address of the President, Professor A. L. Hadgkin, at the Anniversary Meeting, 30 November 1971. Proceedings of the Royal Society, London, A, 326, $\mathbf{v}-\mathbf{x x}$.

Lamb, T. D. \& Simon, E. J. (1976). The relation between intercellular coupling and electrical noise in turtle photoreceptors. Journal of Physiology, London, 263, 257-286.

Levine, M. W. \& Abramov, I. A. (1975). An analysis of spatial summation in the receptive fields of goldfish retinal ganglion cells. Vision Research, 15, 777-789.

Pluvinage, V. \& Green, D. G. (1990). Evidence for a power law intensity code in the coupled cones of the turtle. Vision Research, 30, 673-682.

Robson, J. G. \& Enroth-Cugell, C. (1978). Light distribution in the cat's retinal image. Vision Research, 18, 159-173.

Schnapf, J. L. \& McBurney, R. N. (1980). Light-induced changes in membrane current in cone outer segments of tiger salamander and turtle. Nature, London, 287, 239-241.

Thorson, J. \& Biederman-Thorson, M. (1974). Distribution relaxation process in sensory adaptation. Science, New York, 189, 161-172.

\section{APPENDIX 1}

The model contists of (1) a light distribution function, (2) a Michaelis-Menten relationship between absorbed photons and photocurrent amplitude, and (3) an electrical network of cones (Lamb \& Simon, 1976). Because the slit stimulus generates no electrical gradient parallel to its longitudinal axis, the model is one-dimentional. We define the light distribution function $l_{0} S(x)$ as the light "seen" by a cone situated at a distance " $x$ " from the center of the slit, where $I_{0}$ is a scaling factor and $S(x)$ is a dimensionles function which accounts for the scattering. Since the actual scattering function of the retina is unknown, $S(x)$ is for the moment unspecified.

It is assumed that each cone can be modeled as a current source in parallel with a fixed membrane resistance " $r_{m}$ ". Because cones are electrically coupled the model contains resistances, " $r_{c}$ ", which couples cones to their neighbors. The current source is controlled by light according to a Michaelis-Menten relationship (Schnapf \& MeBurney, 1980):

$$
\frac{i}{i_{\max }}=\frac{I}{I+\sigma}
$$

where " $i$ " is the peak photocurrent, " $i$ mex" is the suturating peak amplitude, " $I$ " is the light intensity falling on the cone, and " $\sigma$ " is the intensity which produces half maximum photocurrent.

A distributed model will first be used to test qualitatively the recruitment hypothesis. On each infinitesimal segment " $\mathrm{d} x$ ", light generates a photocurrent " $i(x) \mathrm{d} x$ " given by:

$$
i(x) \mathrm{d} x \propto \frac{I_{0} S(x)}{I_{0} S(x)+\sigma} \mathrm{d} x .
$$

For a linear coupling, the response amplitude " $V$ " at the origin is proportional to a weighted sum of all the contributions at various distances from the slit:

$$
V \propto \int_{0}^{\infty} W(x) \frac{l_{0}}{l_{0}+\sigma / S(x)} \mathrm{d} x
$$

where $W(x)$ is the weighting function which accounts for the distance dependent coupling [for simplicity $\sigma$ was normalized and $W(x)$ and $S(x)$ were assumed to be symmetrical:

$$
W(x)=W(-x), S(x)=S(-x)]
$$

The question we need to address is: does a function $S(x)$ exist such that equation (Al.3) can be approximated by:

$$
V \cong k \cdot \sqrt{I_{0}}
$$

over the correct range of intensities? If such a function exists, is it a plausible representation of light scatter? As shown in Appendix II, for a light distribution defined analytically by:

$$
S(x) \propto\left\{1+\gamma \int_{0}^{x} W(y) \mathrm{d} y\right\}^{-2} ;
$$

where $\gamma$ is a constant, the slit intensity-response curve will exhibit a linear range at low intensities, a square-root range at medium intensities and a progressive saturation at high intensities.

Since the cone coupling is assumed to be ohmic (Detwiler \& Hodgkin, 1979) the spread of current is described by the equations of a linear leaky cable (Lamb \& Simon, 1976). Then the coupling function $W(x)$ is given by:

$$
W(x) \propto e^{-|x| / \dot{x}}
$$

where $\lambda=\sqrt{r_{m} / r_{s}}$ of the coupling. Substituting into equation (A1.S) we obtain:

$$
S(x) \propto\left[1+\gamma \lambda\left(1-e^{-|x| / \lambda)}\right]^{-2} .\right.
$$

It can be shown that for a slit displaced by a distance $D$ the intensity-response curve also exhibits a power law range but with an exponent which increases with slit displacement, a trend observed in the data. For a full field stimulus $\boldsymbol{S ( x )}$ is constant and (Al.3) reduces to (1), and therefore the intensity-response curve is Michaelis-Menten.

While the distributed model is in qualitative agreement with the data, it is based on a continuous, one-dimensional array of infinitesimally small cones. Thus the analytical solution we derived might nol apply to a discrete array of finite size cones. Furthermore, $S(x)$ is unrealistic since it is too sharp at the origin and has a nonzero asymptotic value. In other words, a more realistic model is needed. A discrete model based on a two dimensional anatomical diatribution of cones and a more realistic light distribution $S(x)$ was developed and numerical simulations were compered with the data. A discrete array of cones was used. The positions of the cones were measured from a turtle retina photomicrograph published by Hodgkin (1971). The positions of 83 red cones, which contain red and orange oil droptets, were digitized. In the model the properties of all receptors with a red pigment were assumed to be identical. 
As in the distributed model, the cones were modeled as current sources and finite membrane resistance interconnected by resistive elements. The current sources were assumed to be controlled by light according to a MichaelisMenten relationship. The mutual resistance between two cones was assumed to be proportional to $K_{0}(d / \delta)$ where $d$ is the distance between the cones, $\delta$ is a constant and $K_{0}($. is a modified Bessel function (Detwiler \& Hodgkin, 1979).

When a slit is flashed all the cones in a band, a $\Delta \mu \mathrm{m}$ in width and parallel to the slit axis, are approximately equally stimulated. Any function $S(x)$ which has in each band an average value approximately equal to the average value of $S(x)$ will produce the same intensity-response curve. Consequently, the function $S(x)$ was modified as follows. Within the range $|x|<5 \mu \mathrm{m}$, it was replaced by a constant $P(0)$. Within the range $5 \mu \mathrm{m}<|x|<50 \mu \mathrm{m}$, the function was kept unchanged except for a scaling factor equal to the ratio $P(0) / S(0)$. For larger values of $x, S(x)$ was multiplied by the damping factor $[1 /(1+a(x-50))$, where " $a$ " is a constant]. This ensured that $S(\infty)=0$. The value $P(0)$, which determines the overall scaling of the distribution, was determined by equating the total light delivered by the slit to that in a $5 \mu \mathrm{m}$ band from a full field stimulus. The resulting piecewise defined function $S^{*}(x)$ is shown on Fig. 9B.

The response $V$ is the sum of the contributions from the coupled cones:

$$
V=\sum_{i} K_{0}\left(d_{i} \delta\right) \frac{I_{0}}{I_{0}+\sigma / S^{*}\left(x_{i}\right)}
$$

where $d_{i}=\left[x_{i}^{2}+y_{i}^{2}\right]^{0.3}$ is the distance between the $i$ th cone and the central cone.

Since equation (A1.8) reduces to a Michaelis-Menten relationship for a full field stimulus $\left[S_{(x)}\right.$ is constant], the parameters $x_{i}$ and $\sigma$ were determined by minimizing the square-error deviations from the full field data points. On double logarithmic coordinates, this corresponds to adjusting the vertical and horizontal positions of all the simulation curves at once, keeping their shapes and relative positions one to another unchanged.

The last free parameter, $\delta$, determines the decrease of response amplitude when the distance between a fixed intensity slit and the receptive field center is increased. Thus, this parameter controls the spacing of the slit simulation curves. By trial and error a value of $18 \mu \mathrm{m}$ was found to provide a good fit.

\section{APPENDIX 2}

One can use the mathematical identity (Thorson \& Biederman-Thorson, 1974);

$$
s^{m} \equiv \frac{1}{\Gamma(m) \Gamma(1-m)} \int_{0}^{x} a^{m-1} \frac{s}{s+a} \mathrm{~d} a .
$$

To satisfy (A1.4), let $m=0.5$ and:

$$
a=1 / S(x)
$$

together with:

$$
W(x) \mathrm{d} x \propto a^{-0.5} \mathrm{~d} a .
$$

Combining (A2.2) and (A2.3), we find:

$$
\frac{\mathrm{d} S^{-0.5}(x)}{\mathrm{d} x} \propto W(x) \text {. }
$$

Finally, by integrating (A2.4):

$$
S(x)=S(0)\left\{1+\gamma \int_{0}^{x} W(y) \mathrm{d} y\right\}^{-2}
$$

where $\gamma$ is a fre parameter. If $W(x)$ is an exponential function (equation Al.6) then:

$$
S(x)=S(0)\left[1+\gamma \lambda\left(1-e^{-|x| / \lambda}\right)\right]^{-2}
$$

In other words, if $S(x)$ and $W(x)$ are related according to (A2.5) and (A1.3), then:

$$
V \propto \int_{a}^{\beta} a^{-0.5} \frac{I_{0}}{I_{0}+a} \mathrm{~d} a
$$

where:

$$
\begin{aligned}
\alpha & =1 / S(0) ; \\
\beta & =1 / S(\infty) \\
& =\alpha(1+\gamma \lambda)^{2} .
\end{aligned}
$$

Solving (A2.7), we find:

$$
V \propto \sqrt{I_{0}}\left(\tan ^{-1} \sqrt{\beta / I_{0}}-\tan ^{-1} \sqrt{\alpha / I_{0}}\right) .
$$

Evaluating equation (A2.10) three ranges of intensities can then be defined:

- Linear range:

If $I_{0} \ll \alpha \ll \beta$; then

$$
V \cong c_{1} I_{0}
$$

where $c_{1}$ is a constant.

- Compressive power law range:

If $\alpha \ll I_{0} \ll \beta$; then

$$
V \cong c_{2} \sqrt{I_{0}}
$$

where $c 2 \cong c 1 \pi \sqrt{\alpha / 2}$.

- Saluration range:

If $\alpha \ll \beta \ll I_{0}$; then

$$
V \simeq c_{3} ;
$$

where the constant $c_{3} \cong c_{1} \sqrt{\alpha \beta}$.

The range of the compressive power law is fixed by the ratio:

$$
\beta / \alpha=(1+\gamma \lambda)^{2} .
$$

According to (A2.12), the ratio $\beta / \alpha$ must be greater than $10^{2}$, the approximate range of the compresaive power law. Fixing arbitrarily $\gamma=1$ and $\lambda=25 \mu \mathrm{m}$ leads to $\beta / \alpha=10^{2.8}$. 